\title{
Correction to: Building Energy Modeling with OpenStudio
}

\author{
Larry Brackney, Andrew Parker, Daniel Macumber, and Kyle Benne
}

\section{Correction to: \\ L. Brackney et al., Building Energy Modeling with OpenStudio, https://doi.org/10.1007/978-3-319-77809-9}

This book was inadvertently published without the online supplementary files for chapters 2 through 9. This has now been updated accordingly.

\footnotetext{
The updated online version of this book can be found at https://doi.org/10.1007/978-3-319-77809-9

The updated online versions of these chapters can be found at https://doi.org/10.1007/978-3-319-77809-9_2 https://doi.org/10.1007/978-3-319-77809-9_3 https://doi.org/10.1007/978-3-319-77809-9_4 https://doi.org/10.1007/978-3-319-77809-9_5 https://doi.org/10.1007/978-3-319-77809-9_6 https://doi.org/10.1007/978-3-319-77809-9_7 https://doi.org/10.1007/978-3-319-77809-9_8 https://doi.org/10.1007/978-3-319-77809-9_9
} 